\title{
Porosity-induced full-range visible-light photodetection via ultrahigh broadband antireflection in $\mathrm{ZnO}$ nanowires
}

\author{
Kapil Gupta ${ }^{1}$, Jr-Ting Lin ${ }^{1}$, Ruey-Chi Wang ${ }^{2}$ and Chuan-Pu Liu ${ }^{1}$
}

This study investigates graded porosity in nanowire arrays to realize a new graded refractive index mechanism that results in unprecedented broadband antireflection and extends the photodetection properties to cover the entire visible-light range. In this work, ZnO nanowires (NWs) are chosen as a model system to demonstrate the porosity-induced antireflection and photodetection properties for visible light. Porous ZnO NW arrays (PZNA) were synthesized by the hydrothermal method followed by controlled hydrogen annealing for different durations. The surface pores of the PZNA were formed with a gradient distribution from the top to the bottom of the nanowires. This pore gradient distribution serves as a new mechanism to achieve a graded refractive index, which provides improved broadband antireflection in PZNA with a minimum reflectance of less than $5 \%$ at $800 \mathrm{~nm}$. Moreover, the cathodoluminescence spectra suggest the evolution of many defects in PZNA, which contribute to defect-state excitation phenomena. Based on their unique features with regard to antireflection, multiple scattering and defect state excitation, the PZNA devices exhibit an exceptional capability for steady photodetection over the entire visible-light range. The corresponding unique photodetection mechanisms for the phenomena are discussed. These new physical phenomena can be readily extended to other 1D materials and used to develop other optoelectronic devices.

NPG Asia Materials (2016) 8, e314; doi:10.1038/am.2016.147; published online 23 September 2016

\section{INTRODUCTION}

Broadband photodetectors are important for many applications, such as air quality monitoring, security communication, flame detection, military surveillance and industrial quality control. ${ }^{1}$ Recent research into photodetectors has mainly focused on achieving a wide spectra response, better stability and high speed, for which nanostructures are widely adopted owing to the freedom of designing various structures and the dimensionality of fulfilling these requirements through various synergistic effects. ${ }^{2}$ However, fabricating semiconductor devices that achieve photodetection with considerable sensitivity over wide spectra remains extremely challenging. To expand the waveleng th range of operation, photodetectors have been designed either by doping or by combining different functional materials. ${ }^{3-6}$ Some of these complicated heterostructures involve complex fabrication processes, which restrict their widespread application. ${ }^{3}$ For example, a multi-step fabrication scheme has been demonstrated for fabricating graphene $/ \mathrm{Ta}_{2} \mathrm{O}_{5}$ /graphene heterostructures for this purpose. ${ }^{6}$ Therefore, the development of a single semiconductor material that can be easily synthesized and can achieve wide spectral sensitivity using a facile fabrication scheme would be extremely valuable.

One key issue involved in photodetectors is the reflection of light at the air-substrate interfaces, which can significantly diminish performance. Antireflection coatings are therefore required to minimize reflections and improve the light collection efficiency for photodetection. One way of improving antireflection is to gradually reduce the refractive index of the film by tuning it from the refractive index of the substrate to the refractive index of air. ${ }^{7}$ Such a graded refractive index can provide broadband antireflection, which can enable the visible light photodetection to cover the entire visible light regime.

Currently, one-dimensional (1D) nanomaterials have attracted significant attention owing to their potential applications in nanoelectronics and optoelectronics. ${ }^{8} \mathrm{ZnO}$, an n-type wide band gap $(\sim 3.37 \mathrm{eV})$ semiconductor with a large exciton binding energy $(\sim 60 \mathrm{meV})$ at room temperature, ${ }^{9}$ is a technologically important material for optoelectronic applications. $\mathrm{ZnO}$ can be easily synthesized in various $1 \mathrm{D}$ nanostructures ${ }^{8-12}$ including nanowires (NWs), ${ }^{13}$ nanotubes $^{14}$ and nanorods; ${ }^{15}$ these structures have also shown promise in various nanoscale electronic and photonic devices, ${ }^{16}$ such as photovoltaic cells, ${ }^{17}$ light-emitting diodes, ${ }^{18}$ gas sensors, ${ }^{19}$ piezoelectric nanogenerators ${ }^{20}$ and photodetectors. ${ }^{21-27}$ However, with regard to photodetection, $\mathrm{ZnO}$ is only considered as a good material for UV photodetection ${ }^{21,23-27}$ owing to its wide band gap, and only a few studies have enabled $\mathrm{ZnO}$ to achieve limited visible light photodetection through doping or heterojunction structures. ${ }^{22,28,29}$ This study is the first to demonstrate a new mechanism for obtaining a porosity-induced graded refractive index, which results in enhanced broadband antireflection properties, can extend the inherent properties of a 1D material, for example, the wide band gap of $\mathrm{ZnO}$ in this case, and can modify the material to enable improved visible-light

${ }^{1}$ Department of Materials Science and Engineering, National Cheng Kung University, Tainan, Taiwan and ${ }^{2}$ Department of Chemical and Materials Engineering, National University of Kaohsiung, Kaohsiung, Taiwan

Correspondence: Professor C-P Liu, Department of Materials Science and Engineering, National Cheng Kung University, No. 1, University Road, Tainan 70101, Taiwan E-mail: cpliu@mail.ncku.edu.tw

Received 14 April 2016; revised 21 July 2016; accepted 29 July 2016 

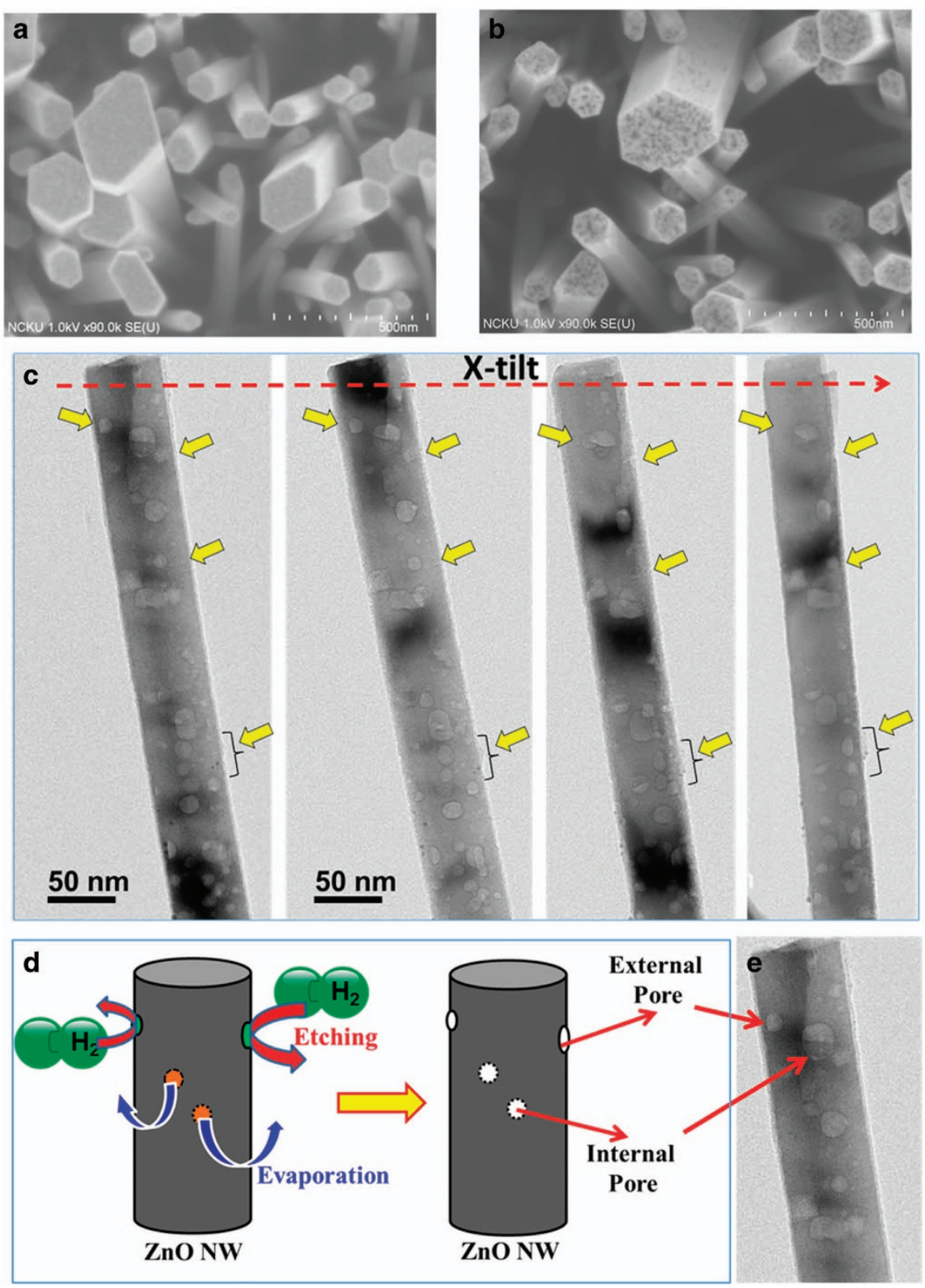

Figure 1 Top-view scanning electron microscopy images of (a) as-grown ZnO nanowires and (b) ZnO nanowires after hydrogen annealing for $3 \mathrm{~h}$. (c) TEM images of PZNA (HA1-ZnO), while the nanowire is rotated about its long axis inside the TEM. The pores that stay fixed at their positions are internal pores, and the pores that move upon rotation (indicated using yellow arrows) are external pores. (d) Schematic of the formation mechanisms of internal and external pores in a ZnO NW upon hydrogen annealing. (e) TEM image of porous ZnO NWs with a $1 \mathrm{~h}$ annealing time (HA1-ZnO), which shows the presence of both internal and external pores.

photodetection over the entire visible-light regime. For this purpose, the $1 \mathrm{D}$ material chosen in this work is $\mathrm{ZnO} \mathrm{NW}$, and porous $\mathrm{ZnO}$ NW arrays (PZNA) were synthesized by a facile fabrication scheme that included controlled hydrogen annealing for different durations.

\section{MATERIALS AND METHODS}

\section{Synthesis of porous $\mathrm{ZnO}$ nanowires}

The schematic diagram of the growth scheme of porous $\mathrm{ZnO} \mathrm{NW}$ arrays (PZNA) is shown in Supplementary Figure S1 of the supporting information. First, the seed layer, a $150 \mathrm{~nm}$ thick $\mathrm{ZnO}$ thin film, was deposited by sputtering on a quartz substrate. Subsequently, $\mathrm{ZnO} \mathrm{NWs}$ were grown by hydrothermal synthesis ${ }^{12,30-32}$ in a 1:1 solution of $0.03 \mathrm{M}$ zinc acetate and $0.03 \mathrm{~m}$ hexamethylenetetramine (HMTA) at $80^{\circ} \mathrm{C}$ for $9 \mathrm{~h}$. The specimen was then rinsed thoroughly in distilled water and dried at $110^{\circ} \mathrm{C}$. Finally, hydrogen annealing was performed to obtain the PZNA, where the as-grown $\mathrm{ZnO} N W$ arrays were annealed at $500{ }^{\circ} \mathrm{C}$ for different durations $(1,2$ and $3 \mathrm{~h})$ in an atmosphere consisting of $\mathrm{Ar}$ and $\mathrm{H}_{2}$ (ratio 80:20) at a flow rate of $10 \mathrm{sccm}$.

\section{Material characterization}

Secondary electron imaging with field-emission scanning electron microscopy (SEM) was performed to observe the morphology of the ZnO NWs using a SU8000 (Hitachi, Tokyo, Japan) operated at $1 \mathrm{kV}$. The optical properties of the as-grown and PZNA samples were characterized at wavelengths between 300 and $800 \mathrm{~nm}$ using a UV-visible spectrophotometer U4100 (Hitachi) equipped with a $60 \mathrm{~mm}$ integrating sphere, and cathodoluminescence spectra (MonoCL4, Gatan Inc., Pleasanton, CA, USA) were obtained. 
a

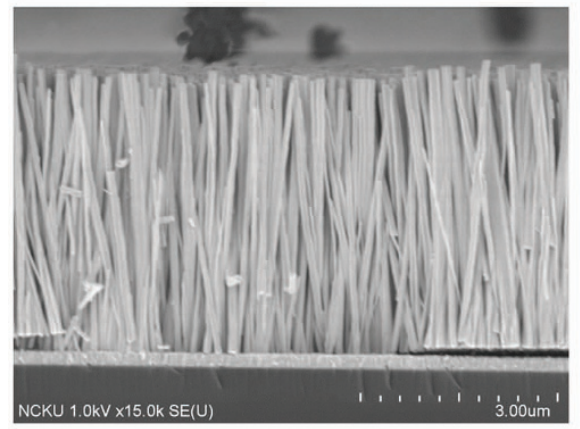

c

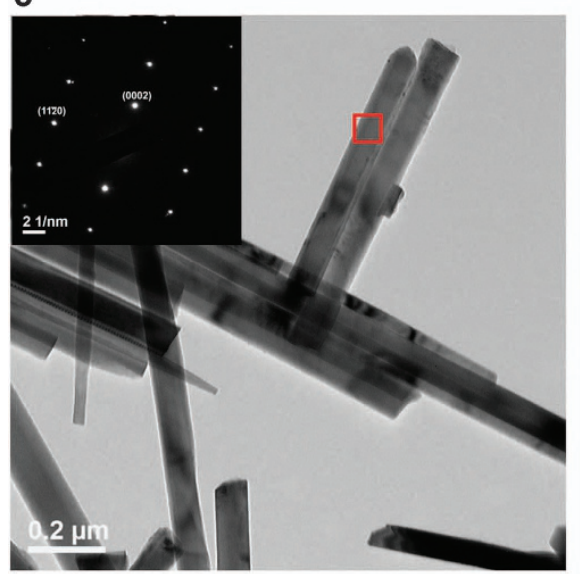

e

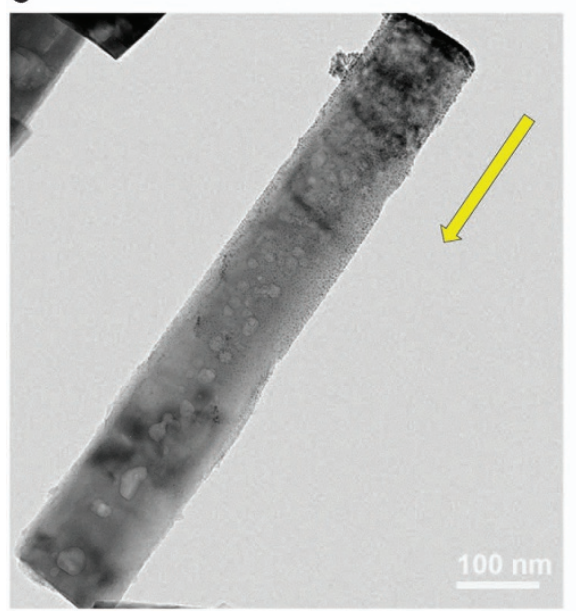

b

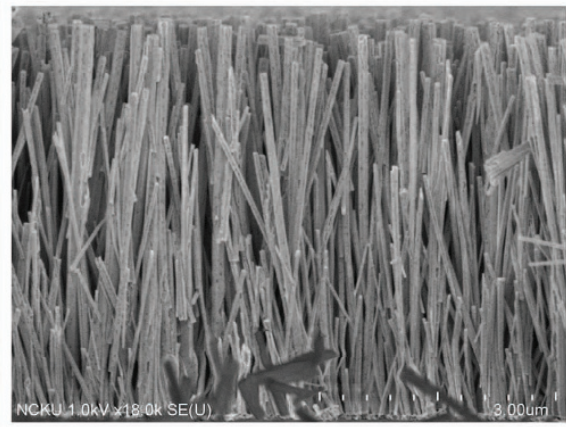

d

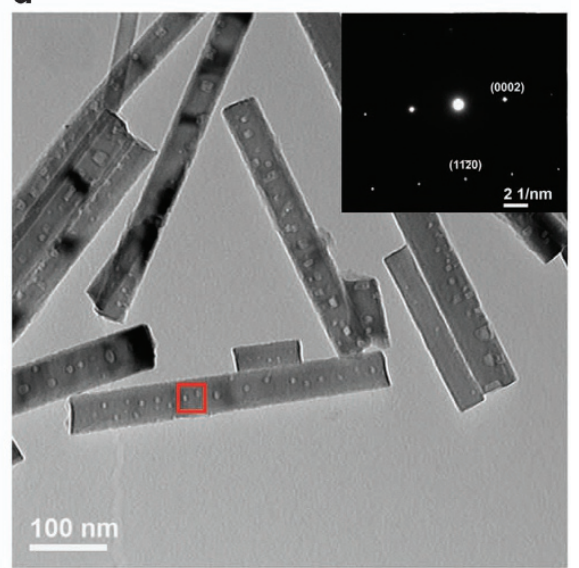

f

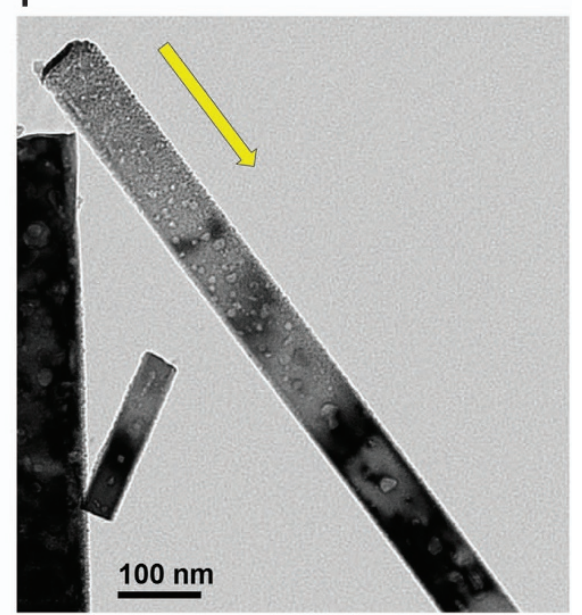

Figure 2 Cross-sectional scanning electron microscopy images of (a) as-grown ZnO nanowires and (b) ZnO nanowires after hydrogen annealing for $3 \mathrm{~h}$. TEM images of (c) as-grown ZnO NWs (U-ZnO), and (d) PZNA (HA1-ZnO), with the insets showing the SAED patterns of the marked regions. (e, f) PZNA $(\mathrm{HA} 1-\mathrm{ZnO})$ with the pore density gradient from the top to bottom of the NWs (the yellow arrows show the top $\rightarrow$ bottom direction). Platinum (Pt) was deposited on top of the film, using an SEM coater as a 'marker', to distinguish between the top and bottom side of the nanowires. Pt particles can be observed on the top portion of the nanowires.

Fabrication and characterization of the visible-light photodetector To fabricate a visible-light photodetector, two Ti/Au $(10 \mathrm{~nm} / 100 \mathrm{~nm})$ electrodes were patterned on top of the as-grown and PZNA samples (the width of the Ti/Au electrodes was $6 \mathrm{~mm}$ and the space between electrodes was $5 \mathrm{~mm}$ ) and then conductive wires were mounted on the electrodes with silver paste. A $5 \mathrm{~V}$ bias voltage was used for the photodetection tests. Light emitting diodes (LEDs) of four different wavelengths covering blue, green, yellow and red light with peak wavelengths at 432.7, 509.3, 597.6 and $644.2 \mathrm{~nm}$, respectively, were employed as the light sources for the photodetection tests. The dependence of the emission wavelength and power of each LED on the input current was measured by an electroluminescence system. Consequently, during the visible-light photodetection tests, the photodetectors were controlled to enable exposure under the same emission power $(1 \mathrm{~mW})$ for different wavelengths of the LEDs, and the induced current change of the photodetectors was recorded at room temperature by an electrical-property measurement unit (Keithley 2400). The spectral profiles of the blue, green, yellow and red LEDs, along with a schematic of the experimental design of the visible-light photodetection test for $\mathrm{ZnO}$ NWs, are shown in Supplementary Figure S2 of the supporting information. 

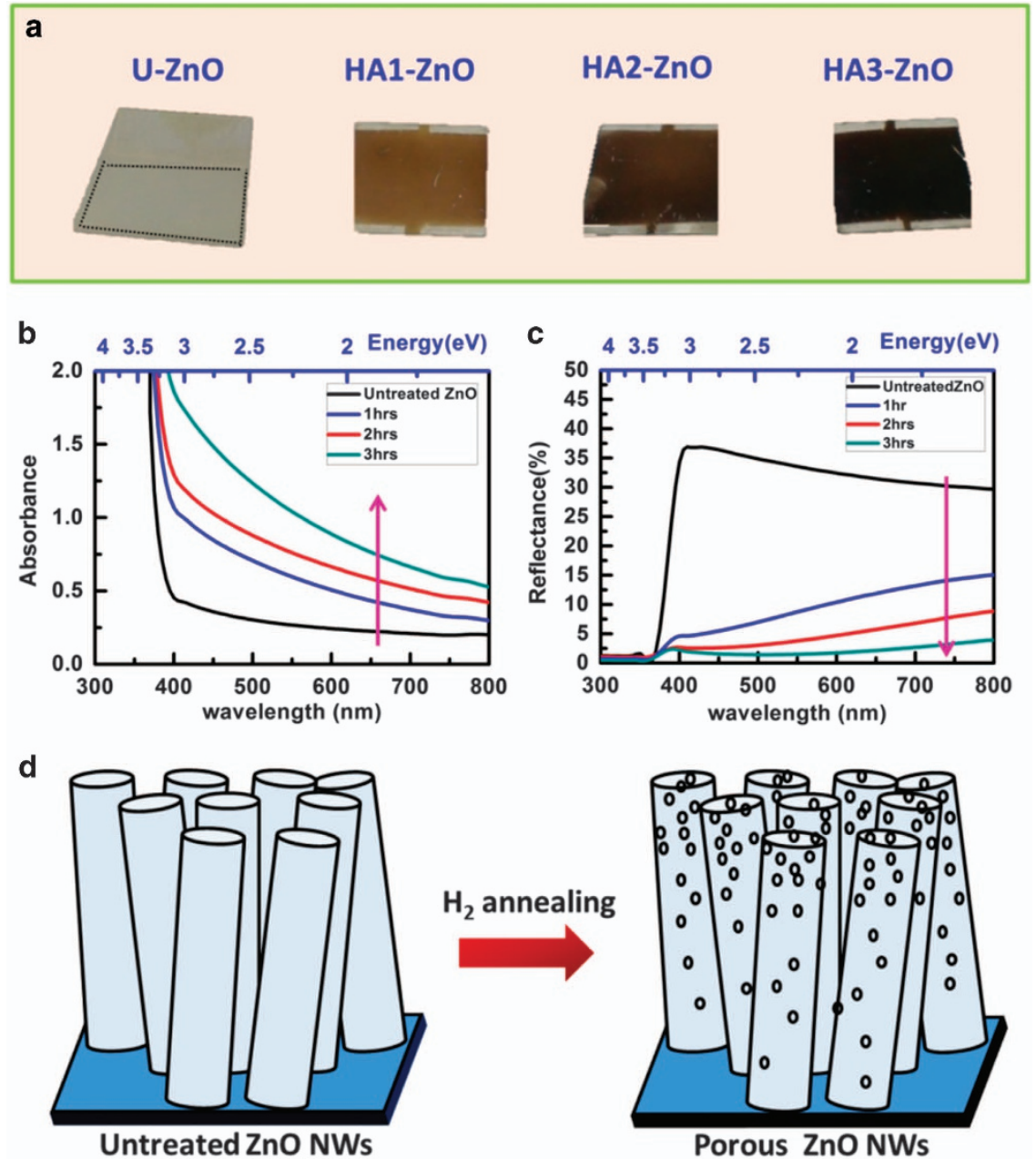

Figure 3 (a) Optical microscopy images of untreated $\mathrm{ZnO}(\mathrm{U}-\mathrm{ZnO})$ and PZNA samples after annealing in hydrogen for $1 \mathrm{~h}(\mathrm{HA} 1-\mathrm{ZnO}), 2 \mathrm{~h}(\mathrm{HA} 2-\mathrm{ZnO})$ and $3 \mathrm{~h}$ (HA3-ZnO). UV-visible (b) absorbance and (c) reflectance spectra of the U-ZnO and PZNA samples after annealing in hydrogen for 1,2 and $3 \mathrm{~h}$. (d) Schematic diagram of porous $\mathrm{ZnO} N W s$ obtained after hydrogen annealing of as-grown $\mathrm{ZnO} N W s$, which shows a gradual decrease in the pore density from the top to bottom of the NWs.

\section{RESULTS AND DISCUSSION}

PZNAs were obtained after hydrogen annealing of as-grown $\mathrm{ZnO}$ NWs, as shown in the schematic diagram of the growth scheme (Supplementary Figure S1 of the supporting information). Top-view and cross-sectional SEM images of the as-grown $\mathrm{ZnO} N W s$ (denoted as untreated $\mathrm{ZnO}, \mathrm{U}-\mathrm{ZnO}$ ) and the PZNA after etching under $\mathrm{H}_{2} / \mathrm{Ar}$ for 1, 2, and $3 \mathrm{~h}$ are shown in Supplementary Figures S3-S5 of the supporting information. According to the SEM and TEM images of the $\mathrm{U}-\mathrm{ZnO}$ NWs and PZNA, the average diameter and length of the $\mathrm{ZnO}$ NWs decreased as the hydrogen annealing time increased, as shown in Supplementary Figure S6a of the supporting information. Furthermore, the surface porosity, defined as the total surface area of the pores divided by the total surface area of the ZnO NWs in an SEM image, increased as the hydrogen annealing time increased, as shown in Supplementary Figure S6b of the supporting information.

The hexagonal columnar morphology of the $\mathrm{ZnO}$ NWs with an average diameter of 70-80 $\mathrm{nm}$ and an average length of $\sim 5 \mu \mathrm{m}$ can be observed from Figure 1a,b and Figure 2a,b, respectively. Figure $1 \mathrm{~b}$ and Figure $2 \mathrm{~b}$ show that the nanopores form on both the top and side surfaces of the $\mathrm{ZnO} \mathrm{NWs}$ after the annealing treatment, with an increasing pore density and a larger density gradient from the top to the bottom for longer treatment times (Supplementary Figures S3-S5 of the supporting information). TEM images of $\mathrm{U}-\mathrm{ZnO}$ and $\mathrm{HAl}-\mathrm{ZnO}$ show that the $\mathrm{ZnO}$ nanowire arrays are single-crystalline, aligned along the $c$-axis and remain unchanged after etching (Figure $2 \mathrm{c}$ and $\mathrm{d}$ ). To study the pore density gradient in the porous nanowires, platinum $(\mathrm{Pt}$ ) was deposited on top of the film using a SEM coater as a 'marker' to distinguish between the top and bottom sides of the nanowires. Thus, the pore density gradient from the top to bottom of the nanowires can be clearly observed from TEM images of PZNA (HA1- $\mathrm{ZnO}$ ), as shown in Figure 2e and f, where yellow arrows show the top $\rightarrow$ bottom direction.

There are two mechanisms responsible for the formation of nanopores in $\mathrm{ZnO}$ NWs upon hydrogen annealing, as shown in the schematic diagram of Figure 1d. External pores form in the $\mathrm{ZnO}$ NWs by direct etching of $\mathrm{ZnO}$ with hydrogen. Moreover, during hydrothermal growth, residues from the organic precursor may be incorporated inside the $\mathrm{ZnO}$ NWs, which can be observed using XPS (Supplementary Figure S7 of the supporting information). ${ }^{33-36}$ As the temperature rises above $450{ }^{\circ} \mathrm{C}$, these precursor residues decompose and evaporate, leaving the nanopores behind and thus forming internal pores. ${ }^{34-36}$ To differentiate between the two types of pores, that is, the internal and external pores, a porous nanowire was rotated by tilting it inside the TEM (Figure 1c). This study was designed based 
on the fact that a pore must be internal if it stays at the same position when a porous nanowire is rotated about its long axis, and must be a surface feature as an external pore if it moves when the nanowire is rotated. As clearly shown in Figure 1c, few pores show significant lateral movement (indicated by the yellow arrows), and these pores can be described as external pores, while other pores are relatively fixed in place, confirming that they are located at a different depth and that they must be internal pores. Therefore, the presence of both external and internal pores can be observed from the TEM images of PZNA (HA1-ZnO), as shown in Figure 1c and e.
Figure 3a shows the optical images of the U-ZnO NWs and PZNA samples after annealing in hydrogen for $1 \mathrm{~h}$ (HA1-ZnO), $2 \mathrm{~h}$ (HA2-ZnO), and $3 \mathrm{~h}$ (HA3-ZnO). It was observed that the color changed dramatically (from white to brown-black) after hydrogen annealing, which indicates the significantly improved antireflection properties of the PZNA upon hydrogen annealing. Hence, to evaluate the effect of the NW surface porosity on the behavior of light traveling through the PZNA samples prepared under different hydrogen annealing conditions, the optical properties of the U-ZnO NWs and PZNA were studied by UV-Visible spectroscopy, with the results
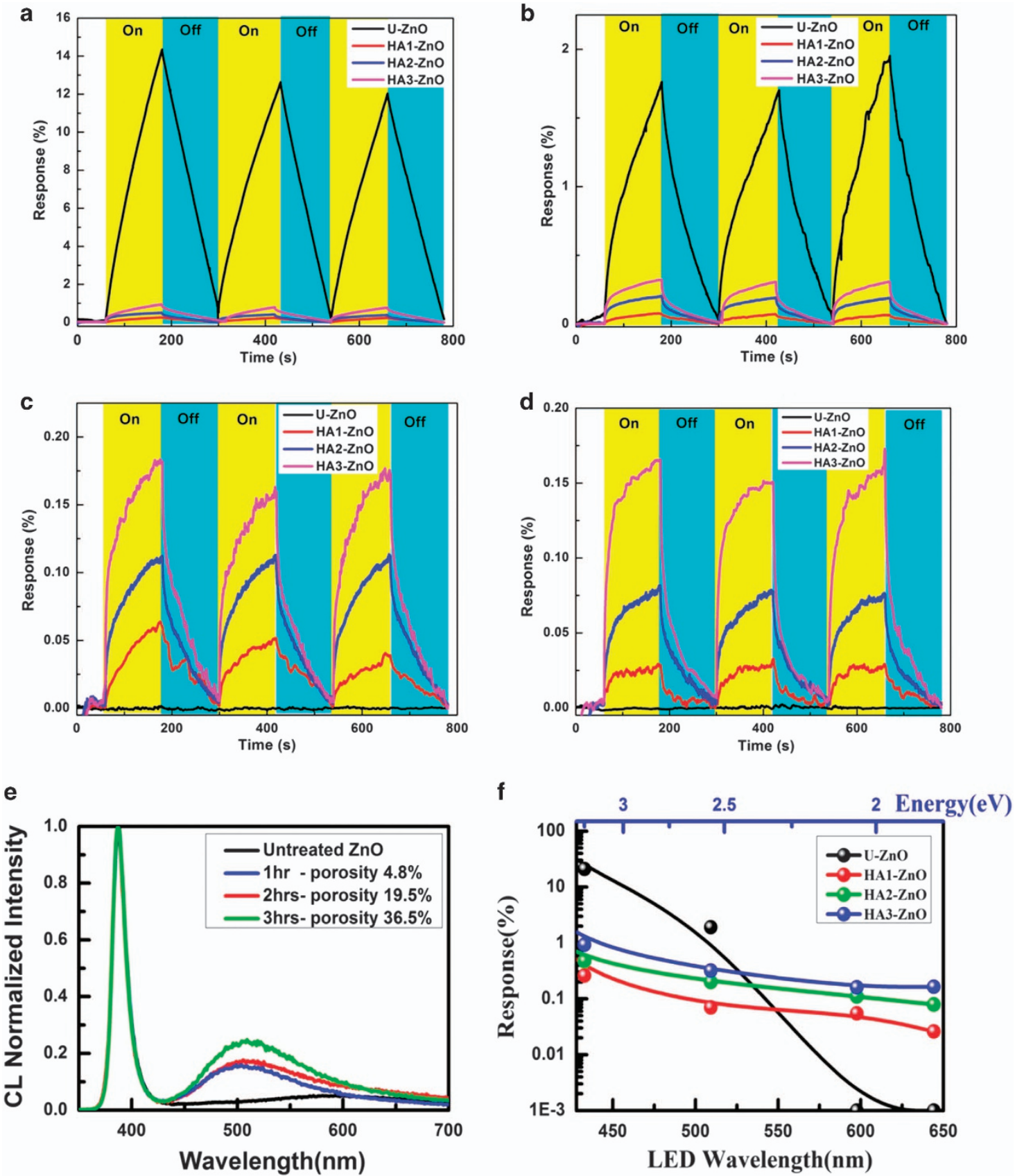

Figure 4 Light response curves of the U-ZnO and all the PZNA devices, after annealing in hydrogen for $1 \mathrm{~h}(\mathrm{HA} 1-\mathrm{ZnO}), 2 \mathrm{~h}(\mathrm{HA} 2-\mathrm{ZnO})$ and $3 \mathrm{~h}(\mathrm{HA} 3-\mathrm{ZnO})$, under exposure to (a) blue LED (b) green LED (c) yellow LED (d) red LED. (e) CL spectra of the untreated ZnO (black) and the PZNA samples after annealing in hydrogen for $1 \mathrm{~h}$ (blue), $2 \mathrm{~h}$ (red) and $3 \mathrm{~h}$ (green), where the SEM is operated at $15 \mathrm{kV}$. (f) Summary of the light responses of U-ZnO and PZNA devices for visible light of all wavelengths. 


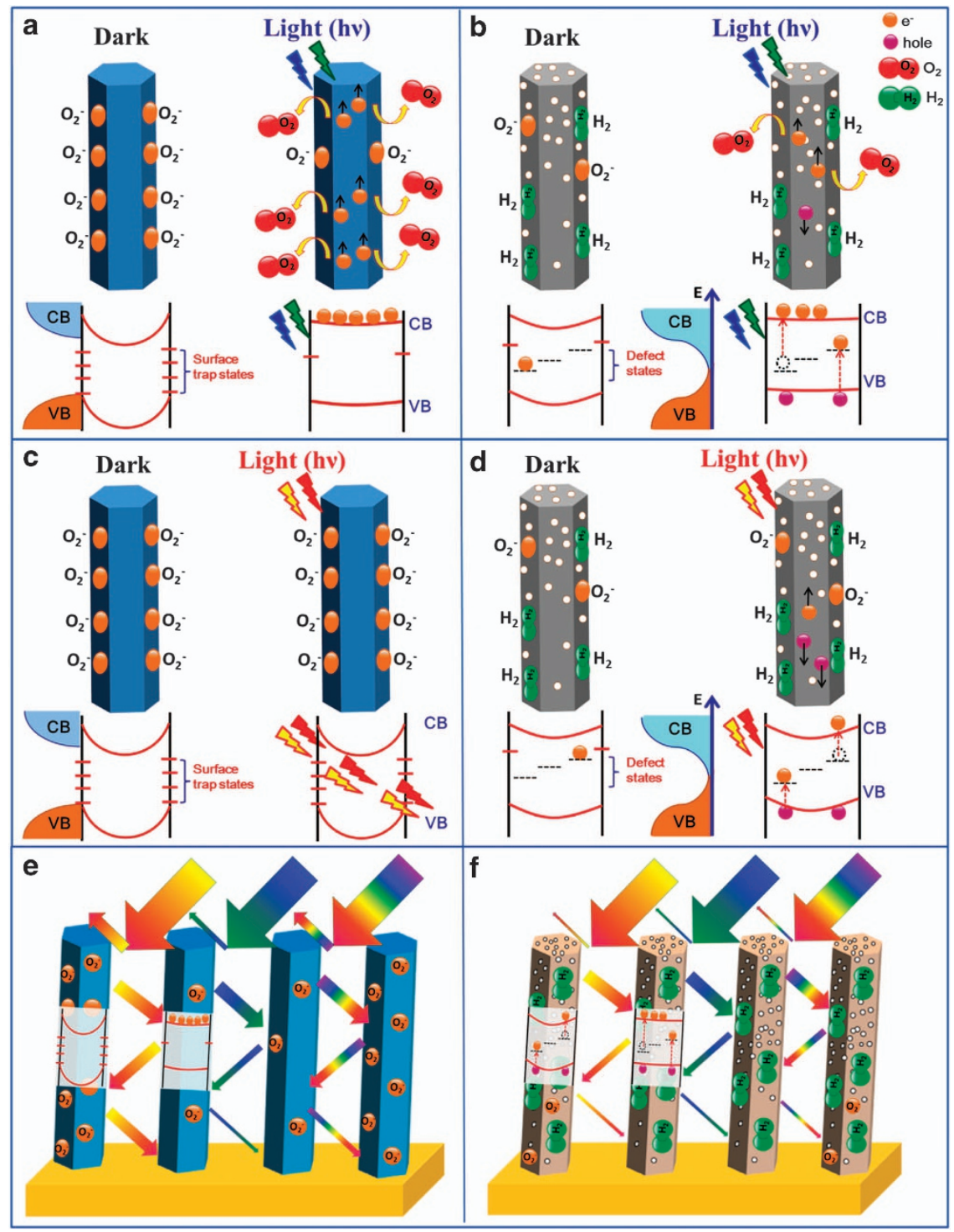

Figure 5 Schematic diagrams along with the energy band illustrations for various photoconduction mechanisms. (a, b) In dark and under blue/green light exposure, which shows that the photon energy breaks the adsorbed oxygen and ZnO bonding for the U-ZnO NWs (a) and PZNA (b). (c, d) In dark and under yellow/red light exposure, which shows the absence of defect state excitation for the U-ZnO NWs (c), and the presence of a continuous band tail and defect state excitations in the PZNA (d). (e, f) Collective phenomena as a result of pore density gradient for ultralow light reflection, porous NW arrays for multiple scattering and the presence (absence) of defect state excitation for the PZNA (U-ZnO NWs). The different band bending behaviors in all the figures is noteworthy.

shown in Figure 3b-c. The absorption spectra in Figure 3b show that the U-ZnO NWs can only absorb UV light and have poor absorption of visible light; however, all the PZNA samples show a significant extension of their optical absorption to the visible-light regime, with longer wavelength absorption for higher-porosity samples, even extending to $800 \mathrm{~nm}$. This result can be attributed to the creation of various point defects by hydrogen etching, which is accompanied by multiple light scattering from the porous NW structures with roughened surfaces that increases the probability of light absorption.

The reflectance spectra are shown in Figure 3c. The results show that the U-ZnO sample has already benefited from the NW configuration, which leads to a low reflectance of $\sim 30-37 \%$ in the entire visible-light regime between $400-800 \mathrm{~nm}$. Strikingly, the nanopores in the PZNA samples further contribute to a decrease in the reflectance when the annealing time is increased; the smallest reflectance of less than $5 \%$, even at $800 \mathrm{~nm}$, is obtained from the PZNA sample with the highest porosity. The ultrahigh broadband antireflection mechanisms with regard to visible light are proposed as follows. At first, the defects created during hydrogen annealing provide enhanced absorption through defect states; these defects contribute little to the extremely low reflectance of PZNA. Moreover, the TEM images in Figure 2e and $\mathrm{f}$ reveal the pore density gradient feature, where the number of pores decreases gradually from the top to bottom of the NWs, as shown in the schematic illustration of Figure $3 \mathrm{~d}$. Since the refractive index of pores (air, $n=1$ ) is less than that of $\mathrm{ZnO}$, a gradient in the density of nanopores will result in a gradual increase in the effective refractive index from the top to the bottom of the NWs. This characteristic of the graded refractive index can result in ultrahigh broadband antireflection. ${ }^{37}$ 
Because the antireflection property is related to the refractive index of the material, ${ }^{38}$ the effective refractive index of the surface is estimated using the density and surface porosity of the NWs, as presented in Supplementary Table S1 of the supporting information. Supplementary Table S1 shows that the surface porosity increases and the effective refractive index decreases, as the hydrogen annealing time increases. The minimum surface refractive index is evaluated to be 1.29 after $3 \mathrm{~h}$ of hydrogen annealing, which is nearly 1.22 , the optimal value of the effective refractive index for optical substrates. ${ }^{39}$

We have thus demonstrated a new porosity-induced mechanism for obtaining a graded refractive index in porous $\mathrm{ZnO} \mathrm{NW}$ arrays, which, along with the improved absorption through defect states, resulted in enhanced antireflection properties that extend to the entire visiblelight region.

In the visible light photodetection test, the response (\%) is defined as the relative change between the dark current $\left(I_{\text {dark }}\right)$ and light current $\left(I_{\text {light }}\right)$, which is given by the following equation:

$$
\text { Response }(\%)=\frac{I_{\text {light }}-I_{\text {dark }}}{I_{\text {dark }}} \times 100 \%
$$

Figure $4 \mathrm{a}-\mathrm{d}$ show the response curves under exposure to different wavelengths of light, that is, blue, green, yellow and red, respectively. Intriguingly, the $\mathrm{U}-\mathrm{ZnO}$ device has a distinct behavior compared with the other PZNA devices in terms of both its shape and the magnitude of its response to different wavelengths. In the blue and green light regimes, the $\mathrm{U}-\mathrm{ZnO}$ device shows a significantly higher response than all the others, while its response diminishes in the yellow and red light regions. Moreover, the $\mathrm{U}-\mathrm{ZnO}$ device requires a significantly longer time for both response $(\sim 110 \mathrm{~s})$ and recovery $(\sim 100 \mathrm{~s})$ in the blue and green light regimes, which can be attributed to the desorption of adsorbed negatively charged oxygen ions that are present on the surface of the U-ZnO NWs in the dark (Figure 5a), and form a low-conductivity depletion layer near the $\mathrm{ZnO} \mathrm{NW}$ surface. ${ }^{21}$ Here, the contribution from photoexcited carriers is negligible, and the desorption and re-adsorption process of oxygen ions is dominant for the $\mathrm{U}-\mathrm{ZnO}$ NWs under blue and green light illumination (Figure $4 \mathrm{a}$ and b), owing to the slow change in the photocurrent over the entire testing period; the process of desorption and re-adsorption of oxygen becomes extinct under illumination with yellow and red light.

To account for the discrepancy in the behavior between the short and long visible-light wavelength illumination for the $\mathrm{U}-\mathrm{ZnO}$ device, we propose that the adsorbed oxygen ions are desorbed by their direct bombardment with incident light photons through energy transfer, as shown in Figure $5 \mathrm{a}^{21}$ Therefore, the cutoff wavelength between green and yellow light represents the bonding energy of oxygen ions on a $\mathrm{ZnO} \mathrm{NW}$ surface at $\sim 2.07-2.43 \mathrm{eV}$, which coincides with a theoretical calculation of $2.47 \mathrm{eV}$ for the $\mathrm{O} / \mathrm{ZnO}$ binding energy. ${ }^{40}$

In contrast, hydrogen annealing for the PZNA samples causes surface passivation with hydrogen by the removal of most of the adsorbed oxygen ions (as shown schematically in Figure 5b), and contributes to the stark contrast in the response curves, where the curves from all the PZNA samples initially experience faster response and recovery for a significant portion of the curves before the curves saturate slowly for all visible-light wavelengths. The response time of $\sim 47 \mathrm{~s}$ for the PZNA with a $3 \mathrm{~h}$ annealing time under exposure to red light is much shorter than the response time of $\sim 110 \mathrm{~s}$ for the as-grown $\mathrm{ZnO} \mathrm{NWs}$ under exposure to blue light (Supplementary Table S3 of the supporting information). Moreover, while all the PZNA devices retain a response (although with a decreasing trend) over the entire visible-light range, the $\mathrm{U}-\mathrm{ZnO}$ device clearly exhibits no response to yellow and red light, as observed in Figure $4 \mathrm{c}$ and $\mathrm{d}$.
The corresponding $I-V$ characteristics are shown in Supplementary Figures S8-S9 of the supporting information.

Therefore, the cathodoluminescence (CL) spectra for all the samples were examined in Figure 4e. From these spectra, it is observed that the defect emissions are almost negligible for the $\mathrm{U}-\mathrm{ZnO}$ sample, which is consistent with the sharp absorption edge observed for the U-ZnO sample in Figure $3 \mathrm{~b}$. Nevertheless, the intensity of the defect emissions at $\sim 500 \mathrm{~nm}$ for the other PZNA samples increases with the annealing time resulting from higher porosity, which is responsible for the higher absorbance in the band-tail states beyond the band gap that extends all the way up to $800 \mathrm{~nm}$ (Figure 3b). Therefore, it seems that hydrogen annealing creates many types of point defects that lead to light absorption and emission at larger wavelengths. Schematic diagrams along with the energy band illustrations of various photoconduction mechanisms are shown in Figure 5. The mechanisms for the $\mathrm{U}-\mathrm{ZnO} \mathrm{NWs}$ under exposure to the blue/green and yellow/red lights are shown in Figure $5 \mathrm{a}$ and $\mathrm{c}$, respectively; the mechanisms for the U-ZnO NWs include the oxygen adsorption-desorption phenomenon (Figure 5a) and the absence of defect states (Figure 5c) in the U-ZnO NWs. Moreover, the mechanisms for the PZNA under exposure to the blue/green and yellow/red lights are shown in Figure 5b and d, respectively; the mechanisms for the PZNA include surface passivation with hydrogen by the removal of most of the adsorbed oxygen ions (in dark), oxygen adsorption-desorption phenomenon and defect state excitation for blue/green light (Figure 5b), and only defect state excitation for yellow/red light (Figure 5d). The existence of the defect states along with the considerable absorbance in the visible-light range for the PZNA samples also confirm the significant response, observed in Figure 4c and $\mathrm{d}$, to illumination by yellow and red light. It is notable that the response for the $\mathrm{U}-\mathrm{ZnO}$ sample in the blue and green light regimes is not due to the defect states but by the desorption of oxygen ions through direct light interactions, as explained earlier.

Finally, the maximum response from each response curve was extracted and plotted in a logarithmic scale as a function of the wavelength in the entire visible-light range for all of the devices in Figure $4 \mathrm{f}$. The corresponding responsivity ( $\mathrm{mA} / \mathrm{W}), \mathrm{EQE}(\%)$, and response time (s) at different wavelengths for the as-grown and PZNA samples are tabulated in Supplementary Table S2 and plotted in Supplementary Figure S10 of the supporting information. Clearly, the PZNA devices exhibit a unique capability of steady photodetection over the entire visible-light range, which is in stark contrast to the $\mathrm{U}-\mathrm{ZnO}$ device that only responds to blue and green light. This incredible photodetection ability of the $\mathrm{ZnO}$-based nanomaterials down to $800 \mathrm{~nm}$ has been derived from the unique structural design described in this work, including the pore density gradient for ultralow light reflection, the porous NW arrays for multiple scattering, and the presence of point defects for light absorption and emission at larger wavelengths. Correspondingly, most of the incident light will penetrate into the porous NW arrays without experiencing losses through surface reflection and then the light will remain inside as a result of multiple scattering events until it is absorbed by various defect states, as shown in Figure $5 \mathrm{e}$ and $\mathrm{f}$. Therefore, we demonstrate a new mechanism for obtaining a porosity-induced graded refractive index, which results in enhanced broadband antireflection properties that can transform a wide bandgap 1D material (that is, $\mathrm{ZnO} \mathrm{NWs}$ ) into a full-range visible-light photodetector.

In summary, we have successfully synthesized porous $\mathrm{ZnO}$ NWs by using a hydrothermal method followed by hydrogen annealing. The surface porosity was controlled by the hydrogen annealing time. The PZNA with the highest porosity showed broadband antireflection, 
with a reflectance of less than $5 \%$ at wavelengths between 400 and $800 \mathrm{~nm}$. This broadband antireflection is caused primarily by the porosity-induced graded refractive index and partly by the defect-related absorption and the effects of multiple scattering. The different phenomena, including antireflection, multiple scattering and defect emissions, work synergistically to enhance the visible-light photodetection over the entire visible-light regime. These new physical phenomena can be readily extended to other 1D materials and used to develop empower other optoelectronic devices.

\section{CONFLICT OF INTEREST}

The authors declare no conflict of interest.

\section{ACKNOWLEDGEMENTS}

All authors contributed to the discussions and manuscript revision. This work was supported by the Ministry of Science and Technology (MOST) of Taiwan under grant MOST102-2923-E-006-003-MY3. The authors thank the Center for Micro/Nano Science and Technology, National Cheng Kung University, for providing equipment and technical support.

Author contributions: JL and CL conceived the project. JL carried out the synthesis of the material, and performed the characterization studies. KG carried out TEM studies. KG and CL wrote the manuscript. RW and CL supervised the research work.

1 Razeghi, M. \& Rogalski, A. Semiconductor ultraviolet detectors. J. Appl. Phys. 79, 7433-7473 (1996)

2 Zhai, T., Li, L., Wang, X., Fang, X., Bando, Y. \& Golberg, D. Recent Developments in One-Dimensional Inorganic Nanostructures for Photodetectors. Adv. Funct. Mater. 20, 4233-4248 (2010).

3 Hsu, C.-Y., Lien, D.-H., Lu, S.-Y., Chen, C.-Y., Kang, C.-F., Chueh, Y.-L., Hsu, W.-K. \& He, J.-H. Supersensitive, Ultrafast, and Broad-Band Light-Harvesting Scheme Employing Carbon Nanotube $/ \mathrm{TiO}_{2}$ Core-Shell Nanowire Geometry. ACS Nano 6 , 6687-6692 (2012)

4 Wei, D., Liu, Y., Cao, L., Zhang, H., Huang, L. \& Yu, G. Synthesis and Photoelectric Properties of Coaxial Schottky Junctions of ZnS and Carbon Nanotubes. Chem. Mat. 22, 288-293 (2010)

5 Qiao, H., Yuan, J., Xu, Z., Chen, C., Lin, S., Wang, Y., Song, J., Liu, Y., Khan, Q., Hoh, H. Y., Pan, C.-X., Li, S. \& Bao, Q. Broadband Photodetectors Based on Graphene- $\mathrm{Bi}_{2} \mathrm{Te}_{3}$ Heterostructure. ACS Nano 9, 1886-1894 (2015).

6 Liu, C.-H., Chang, Y.-C., Norris, T. B. \& Zhong, Z. Graphene photodetectors with ultra-broadband and high responsivity at room temperature. Nat. Nanotechnol. 9, 273-278 (2014).

7 Raut, H. K., Ganesh, V. A., Nair, A. S. \& Ramakrishna, S. Anti-reflective coatings: A critical, in-depth review. Energ. Environ. Sci. 4, 3779-3804 (2011).

8 Zhong Lin, W. Zinc oxide nanostructures: growth, properties and applications. J. Phys. Condens. Matter 16, R829 (2004).

9 Özgür, Ü., Alivov, Y. I., Liu, C., Teke, A., Reshchikov, M. A., Doğan, S., Avrutin, V., Cho, S.-J. \& Morkoç, H. A comprehensive review of $\mathrm{ZnO}$ materials and devices. J. Appl. Phys. 98, 041301 (2005).

10 Fan, Z. \& Lu, J. G. Zinc oxide nanostructures: synthesis and properties. J. Nanosci. Nanotechno. 5, 1561-1573 (2005).

11 Jayadevan, K. P. \& Tseng, T. Y. One-dimensional ZnO nanostructures. J. Nanosci. Nanotechno. 12, 4409-4457 (2012).

$12 \mathrm{Xu}, \mathrm{S}$. \& Wang, Z. One-dimensional ZnO nanostructures: Solution growth and functional properties. Nano Res. 4, 1013-1098 (2011).

13 Yang, P. D., Yan, H. Q., Mao, S., Russo, R., Johnson, J., Saykally, R., Morris, N., Pham, J., He, R. \& Choi, H. J. Controlled growth of $\mathrm{ZnO}$ nanowires and their optical properties. Adv. Funct. Mater. 12, 323 (2002).

14 Kong, X. Y., Ding, Y. \& Wang, Z. L. Metal-semiconductor Zn-ZnO core-shell nanobelts and nanotubes. J. Phys. Chem. B 108, 570 (2004).

15 Vayssieres, L. Growth of arrayed nanorods and nanowires of $\mathrm{ZnO}$ from aqueous solutions. Adv. Mater. 15, 464 (2003).

16 Chu, S., Wang, G., Zhou, W., Lin, Y., Chernyak, L., Zhao, J., Kong, J., Li, L., Ren, J. \& Liu, J. Electrically pumped waveguide lasing from ZnO nanowires. Nat. Nanotechnol. 6, 506-510 (2011).

17 Law, M., Greene, L. E., Johnson, J. C., Saykally, R. \& Yang, P. Nanowire dye-sensitized solar cells. Nat. Mater. 4, 455-459 (2005).

18 Bao, J., Zimmler, M. A., Capasso, F., Wang, X. \& Ren, Z. F. Broadband ZnO single-nanowire light-emitting diode. Nano Lett. 6, 1719-1722 (2006).
19 Pan, X., Liu, X., Bermak, A. \& Fan, Z. Self-gating effect induced large performance improvement of ZnO nanocomb gas sensors. ACS Nano 7, 9318-9324 (2013).

20 Xu, S., Qin, Y., Xu, C., Wei, Y., Yang, R. \& Wang, Z. L. Self-powered nanowire devices. Nat. Nanotechnol. 5, 366-373 (2010).

21 Soci, C., Zhang, A., Xiang, B., Dayeh, S. A., Aplin, D. P. R., Park, J., Bao, X. Y., Lo, Y. H. \& Wang, D. ZnO Nanowire UV photodetectors with high internal gain. Nano Lett. 7, 1003-1009 (2007).

22 Zhan, Z., Zheng, L., Pan, Y., Sun, G. \& Li, L. Self-powered, visible-light photodetector based on thermally reduced graphene oxide-ZnO ( $\mathrm{rGO}-\mathrm{ZnO})$ hybrid nanostructure. J. Mater. Chem. 22, 2589-2595 (2012).

23 Chaaya, A. A., Bechelany, M., Balme, S. \& Miele, P. ZnO 1D nanostructures designed by combining atomic layer deposition and electrospinning for UV sensor applications. J. Mater. Chem. A 2, 20650-20658 (2014).

24 Kind, H., Yan, H., Messer, B., Law, M. \& Yang, P. Nanowire ultraviolet photodetectors and optical switches. Adv. Mater. 14, 158-160 (2002).

25 Junya, S., Nobutaka, N., Shin-ichiro, H., Makoto, U., Kiminobu, I., Mitsuhiro, H., Tatsuo, O. \& Masanori, H. Dielectrophoretic fabrication and characterization of a $\mathrm{ZnO}$ nanowire-based UV photosensor. Nanotechnology 17, 2567 (2006).

26 Cheng, G., Wu, X., Liu, B., Li, B., Zhang, X. \& Du, Z. ZnO nanowire Schottky barrier ultraviolet photodetector with high sensitivity and fast recovery speed. Appl. Phys. Lett. 99, 203105 (2011).

27 Liu, X., Gu, L., Zhang, Q., Wu, J., Long, Y. \& Fan, Z. All-printable band-edge modulated ZnO nanowire photodetectors with ultra-high detectivity. Nat. Commun. 5, 4007 (2014).

28 Zheng, Z., Gan, L., Li, H., Ma, Y., Bando, Y., Golberg, D. \& Zhai, T. A fully transparent and flexible ultraviolet-visible photodetector based on controlled electrospun $\mathrm{ZnO}-\mathrm{CdO}$ heterojunction nanofiber arrays. Adv. Funct. Mater. 25, 5885-5894 (2015).

29 Zhu, L., Li, C., Li, Y., Feng, C., Li, F., Zhang, D., Chen, Z., Wen, S. \& Ruan, S. Visible-light photodetector with enhanced performance based on a ZnO@CdS heterostructure. J. Mater. Chem. C 3, 2231-2236 (2015).

30 Fang, X., Li, J., Zhao, D., Shen, D., Li, B. \& Wang, X. Phosphorus-doped p-type $\mathrm{ZnO}$ nanorods and $\mathrm{ZnO}$ nanorod $\mathrm{p}-\mathrm{n}$ homojunction LED fabricated by hydrothermal method. J. Phys. Chem. C 113, 21208-21212 (2009).

31 Sohn, J. I., Jung, Y.-I., Baek, S.-H., Cha, S., Jang, J. E., Cho, C.-H., Kim, J. H., Kim, J. M. \& Park, I.-K. A low temperature process for phosphorous doped $\mathrm{ZnO}$ nanorods via a combination of hydrothermal and spin-on dopant methods. Nanoscale 6 , 2046-2051 (2014).

32 Lee, J., Cha, S., Kim, J., Nam, H., Lee, S., Ko, W., Wang, K. L., Park, J. \& Hong, J. p-Type conduction characteristics of lithium-doped $\mathrm{ZnO}$ nanowires. Adv. Mater. 23, 4183-4187 (2011).

33 Lee, S. H., Lee, H. J., Goto, H., Cho, M.-W. \& Yao, T. Fabrication of porous ZnO nanostructures and morphology control. Phys. Status Solidi C 4, 1747-1750 (2007).

34 Ghatak, J., Huang, J.-H., Huang, P.-C., Shih, Y.-I. \& Liu, C.-P. Synthesis of porous single crystalline $\mathrm{ZnO}$ nanowires and the derivation of surface free energy from equilibrium nanopore. J. Electrochem. Soc. 159, H239-H242 (2012).

35 Govender, K., Boyle, D. S., Kenway, P. B. \& O'Brien, P. Understanding the factors that govern the deposition and morphology of thin films of $\mathrm{ZnO}$ from aqueous solution. J. Mater. Chem. 14, 2575-2591 (2004).

36 Park, S. K., Park, J. H., Ko, K. Y., Yoon, S., Chu, K. S., Kim, W. \& Do, Y. R. Hydrothermal-electrochemical synthesis of ZnO nanorods. Cryst. Growth Des. 9, 3615-3620 (2009)

37 Perl, E. E., McMahon, W. E., Farrell, R. M., DenBaars, S. P., Speck, J. S. \& Bowers, J. E. Surface structured optical coatings with near-perfect broadband and wide-angle antireflective properties. Nano Lett. 14, 5960-5964 (2014).

38 Aurang, P., Demircioglu, O., Es, F., Turan, R. \& Unalan, H. E. ZnO nanorods as antireflective coatings for industrial-scale single-crystalline silicon solar cells. J. Am. Ceram. Soc. 96, 1253-1257 (2013).

39 Guldin, S., Kohn, P., Stefik, M., Song, J., Divitini, G., Ecarla, F., Ducati, C., Wiesner, U. \& Steiner, U. Self-cleaning antireflective optical coatings. Nano Lett. 13, 5329-5335 (2013).

40 Breedon, M., Spencer, M. J. S. \& Yarovsky, I. Adsorption of atomic nitrogen and oxygen on $\mathrm{ZnO}(2-1-10)$ surface: a density functional theory study. J. Phys. Condens. Matter 21, 144208 (2009).

(c) (i) This work is licensed under a Creative Commons Attribution 4.0 International License. The images or other third party material in this article are included in the article's Creative Commons license, unless indicated otherwise in the credit line; if the material is not included under the Creative Commons license, users will need to obtain permission from the license holder to reproduce the material. To view a copy of this license, visit http:// creativecommons.org/licenses/by/4.0/

(C) The Author(s) 2016

Supplementary Information accompanies the paper on the NPG Asia Materials website (http://www.nature.com/am) 